ISSN electrónico: 2172-9077

DOI: https://doi.org/10.14201/fjc-v22-22695

\title{
ImPaCtO DE LOS INFLUENCERS EN LAS Estrategias Promocionales del Sector Gastronómico de la CiUdad de Medellín
}

\section{The Impact of Influencers on the Promotional Strategies of the Gastronomic Sectors in Medellín}

\author{
Sandra Milena AMAYA HENAO \\ Administrador Comercial Egresada del Tecnológico de Antioquia, \\ Institución Universitaria. Medellín, Colombia. \\ E-mail: sammy7022@hotmail.com \\ (iD) ORCID: https://orcid.org/0000-0003-4029-304X
}

\section{Germán Augusto SILVA CORTÉS}

Administrador Comercial Egresado del Tecnológico de Antioquia, Institución Universitaria. Medellín, Colombia.

E-mail: germansc82@gmail.com

(iD) ORCID: https://orcid.org/0000-0003-3363-8898

\section{Yanyn Aurora RINCÓN QUINTERO}

Ph.D. Docente e Investigadora del Tecnológico de Antioquia, Institución Universitaria. Medellín, Colombia.

E-mail: yanyn.rincon@gmail.com

(iD) ORCID: https://orcid.org/0000-0003-2427-3161

Fecha de recepción del artículo: 14/03/2020

Fecha de aceptación definitiva: 30/03/2020

\begin{abstract}
RESUMEN
Los Influencers gastronómicos se han constituido en figuras que inciden altamente en el reconocimiento e impulso de los negocios en este sector y a su vez permiten a la comunidad conocer la diversidad gastronómica que puede encontrarse en la ciudad de Medellín. Este artículo tiene como objetivo conocer el impacto de los influencers en las estrategias promocionales del sector gastronómico de la ciudad de Medellín, por medio de una investigación de tipo descriptiva, a partir de un diseño no experimental, transeccional descriptiva y de campo; empleando como técnicas para la recolección de datos la entrevista y la encuesta a través de un cuestionario multivariado, de opciones múltiples. Los resultados mostraron que los influencers son la opción predilecta cuando los establecimientos del sector desean promocionar sus productos, ya que logran un mayor alcance en menos tiempo y a menor costo que con otros medios más tradicionales, particularmente desde la red social Instagram; además, de contribuir en gran medida al aumento en los indicadores de cumplimiento en ventas gracias a su intervención. Se concluye que
\end{abstract}


el impacto del influencer en las estrategias promocionales para el sector gastronómico de la ciudad de Medellín es altamente favorable.

Palabras clave: Influencers; impacto; estrategias promocionales; sector gastronómico.

\begin{abstract}
Gastronomic influencers have become figures that highly influence the recognition and promotion of business in this sector and, in turn, allow the community to know the gastronomic diversity that can be found in the city of Medellin. This article aims to know the impact of influencers on the promotional strategies of the gastronomic sector of the city of Medellin, via descriptive research, based on a non-experimental, descriptive and field-based transectional design; us-ing as techniques for data collection the interview and the survey through a multivariate, or multiple options question-naire. The results showed that influencers are the preferred option when establishments in the sector want to promote their products, since they have a greater reach in less time and at a lower cost than with other more traditional means, particularly from the social network Instagram; in addition, they contribute greatly to the increase in sales compliance indicators thanks to their intervention. It is concluded that the impact of influencers on promotional strategies for the gastronomic sector of the city of Medellín is highly positive.
\end{abstract}

Keywords: Influencers; impact; promotional strategies; gastronomy sector.

\title{
1. Introducción
}

Al estar inmersos en una era globalizada donde se accede a la información de manera más inmediata y en tiempo real, hay que preguntarse acerca de cómo todos estos canales de información, como blogs, redes sociales, prensa digital, webs corporativas, entre otros, han afectado y modificado las formas de comunicar la marca y sus propuestas de valor hacia sus mercados objetivo las organizaciones y empresas, y en este proceso se ha podido detectar que están empleando personalidades influyentes en las diferentes plataformas digitales, en el ánimo de interactuar de forma más directa con los clientes actuales y futuros, transmitiendo mayor credibilidad en sus mensajes incluso que con otros medios promocionales tradicionales, en un esfuerzo colaborativo donde las organizaciones llegan a su público objetivo y los llamados influencers generan mayor impacto con el contenido compartido en sus redes sociales.

En las últimas dos décadas, con la masificación del uso del internet y de la aparición y desarrollo de las redes sociales, se ha visto, como éstas poco a poco han ido convirtiéndose en una herramienta comunicativa cada vez más extensa y poderosa; además, con el auge de dispositivos móviles, cada vez más sofisticados, han constituido una combinación que ha sabido ser aprovechada por grandes empresas para el impulso de sus productos a través de estrategias promocionales que posibiliten el aumento de su participación en el mercado a través de estos medios.

La modalidad testimonial es uno de los medios que las marcas han adoptado para influenciar a los usuarios y consumidores en la adopción de los productos-servicios asociados a sus marcas, donde una figura reconocida a través de su opinión y de su profesión brindan testimonio de emplear productos y servicios para captar mayor número de usuarios o consumidores; es entonces, que usando el poder de influenciar por medio de la palabra y el testimonio de uso y mediante las redes sociales existentes como aparecen los llamados Influencers; una comunidad de personas altamente 
aceptadas y seguidas, que logran transmitir sus ideas, opiniones y estilos de vida en las diferentes redes.

Las marcas se han dado cuenta del potencial que tienen estos Influencers, personas que consumen, generan información, con cierta experticia en un tema en específico e interactúan y comparten sus opiniones, e ideas con sus seguidores, (Dorantes, 2012) para poder aprovecharlos y hacer llegar a través de ellos sus productos o servicios a cada vez más personas y lugares, ya que por medio de sus opiniones pueden mejorar y aumentar la reputación y recordación de su marca.

En el sector gastronómico, se puede vislumbrar como personalidades como Anthony Bourdain (1956-2018) y Andrew Zimmern (1961), afamados y reconocidos Chefs, nacidos en Nueva York, quienes por medio de la gastronomía, acercaron diversas culturas y sociedades del mundo impulsando el sector gastronómico, sugiriendo visitar y conocer destinos gastronómicos y establecimientos a través de los sabores propios de cada cultura en aquellos destinos que en algún momento visitaron, donde han mostrado al mundo los platos más extraños, con los ingredientes más inusuales, que puedan prepararse allí, describiendo meticulosamente cada detalle en su preparación, ingredientes y sabores. Todo esto en programas televisivos como Cook's Tour del canal Food Network, No Reservations en Travel Channel (2005-2012) o Parts Unknown entre los años 2013-2018 ABC (2018) y programa Bizarre Foods o como se le conoce en Latinoamérica Comidas Exóticas, transmitido por el canal Discovery Travel E Living desde el año 2007, presentado por Zimmern (Travelchannel, 2019).

La gastronomía de una región es la esencia y reflejo de la cultura de una comunidad, un pueblo o un país, le da identidad y está estrechamente ligada a su historia, 'influenciando a través de generaciones', según Meléndez y Cañez (2010), el resultado de lo que llega a la mesa de todo comensal. La creación y fusión de diferentes conceptos gastronómicos, le da valor agregado a dicha cultura y proporciona la oportunidad de tener momentos para compartir con otras personas alrededor de una buena mesa, genera la curiosidad de probar algo nuevo bien sea desde lo tradicional hasta lo exótico, propiciando así el desarrollo de diversidad de encuentros familiares, sociales o de negocios.

El desarrollo tecnológico y las redes sociales han posibilitado que los establecimientos del sector gastronómico encuentren nuevas maneras de hacer que sus productos lleguen a mayor número de personas, y así mismo, generar en sus posibles nuevos clientes la promesa de una experiencia memorables y es allí, donde los comensales esperan encontrar ciertas bondades o características propias de la experiencia que quieren tener allí donde lleguen, como por ejemplo y solo por citar algunos, atención correcta, rápida y concentrada, buenas recomendaciones y completas, trato amable y sincero, anticipación y prevención, comprensión y tolerancia. (Höchsmann, 2014).

Por otra parte, los influencers son amantes de las plataformas públicas, no sólo virtuales y sino también reales, siendo particularmente estos últimos espacios propicios para la interacción y socialización de éstos con su público objetivo en un espacio físico real y generando aún más credibilidad en sus testimonios. Los influencers al ser figuras públicas pueden aprovechar esa condición para convocar mayor afluencia de comensales en aquellos lugares que visitan. Los restaurantes suelen tener mayor afluencia de clientes cuando sus dueños son figuras públicas o cuando quienes los frecuentan tienen esa misma condición de personalidad reconocida y cuando las personas saben que pueden encontrar estas personalidades que reconocen, siguen o 
incluso admiran, los aprovechan para socializar con ellos y así se propician espacios para llevan a cabo relaciones públicas por parte de quienes comparten e interactúan en aquellos esos espacios.

Todo lo anterior, permite vislumbrar cómo el sector gastronómico de la ciudad de Medellín, al considerar la aparición de influencers en este nicho de mercado, se ha convertido en un sector impulsado por medio de las recomendaciones, reseñas e invitaciones para degustar un sin fin de lugares novedosos y tradicionales, creando mayor flujo de comensales en establecimientos antes poco o nada conocidos y generando mayores ingresos para estos establecimientos, lo que permite orientar la presente investigación a partir la necesidad de 'Determinar el impacto del Influencer en las estrategias promocionales para el sector gastronómico de la ciudad de Medellín'.

Dado que en esta era de la globalización y digitalización de la información el mundo está cambiando permanentemente con más ímpetu que nunca y las organizaciones se ven en la necesidad de buscar la redefinición de sus estrategias de comunicación, particularmente en ser más notorios en ambientes digitales, enfatizando en las redes sociales. Sin embargo, con la gran cantidad de plataformas digitales y redes sociales existentes, las organizaciones se ven exigidas a ser más competitivas a la hora de desarrollar estas nuevas estrategias para lograr conectarse de manera más acertada con su mercado objetivo. Además, la toma de decisiones de los consumidores y los hábitos de consumo han ido evolucionando debido a como se les transmite la información y también de cómo la comparten en internet.

Con la aparición de los influencers se ve también cómo emergen nuevas tendencias donde las organizaciones tienen la responsabilidad de planear y gestionar correctamente sus estrategias para poder participar de manera más activa en los entornos sociales, ya que los consumidores actuales son cada vez más exigentes y tienen la capacidad de valorar con mayor precisión los pro y los contra al momento de llevar a cabo la elección de diferentes tipos de productos y servicios, pues tienen mayor capacidad de acceder a la información por sus propios medios.

Los influencers han ido convirtiéndose en elementos que permiten a las organizaciones identificar nuevos entornos donde puedan expandirse comercialmente y elaborar estrategias de mercadeo que les posibiliten intervenir y aprovechar las dinámicas actuales de consumo.

La presente investigación ha sido completada en el año 2019; delimitando su área a la variable influencer y estructurando en torno al sector gastronómico de la ciudad de Medellín ya que actualmente dicho sector ha propiciado la aparición en escena de diversas cuentas en redes sociales, particularmente Instagram, de personas que han constituido la figura de influencer y que en un principio muchos de ellos lo realizaban como un simple bobby y con el paso del tiempo fue constituyéndose propiamente en una profesión que les llevó incluso a agruparse o agremiarse para ofrecer paquetes de servicios con los que promocionan marcas y productos de los establecimientos del sector e incluso en algunos casos su conocimiento y experticia les llevó a crear sus propias agencias de marketing gastronómico. Para esta investigación se tomaron como muestra para el desarrollo de la misma algunas de las cuentas de influencers gastronómicos más reconocidas de la ciudad de Medellín como @Dondecomer, @ QuehacerMedellín, @FoodguideMedellin y @Gastronomiacasual.

Teóricamente se circunscribe a la línea de investigación de gestión y estudios organizacionales adscrita al grupo Research Enterprise Development (RED), de la Facultad de Ciencias Administrativas y Económicas del Tecnológico de Antioquia, 
Institución Universitaria y están soportados en autores como Pujol (1999), Kotler, Cámara, Grande \& Cruz (2000), Tellis \& Redondo (2002), Burnett (1996), Boyd \& Elison (2008), Celaya (2008), Dorantes (2012), con quienes se fundamentaron las bases teórico conceptuales claves para el desarrollo de la presente investigación.

\section{Objetivos e Hipótesis}

La presente investigación se orienta a partir de la pregunta problémica: ¿De qué manera impactan los influencers las estrategias promocionales del sector gastronómico en la ciudad de Medellín?, lo que establece como supuesto implícito para quienes investigan que el uso de Influencers resulta favorable en las estrategias promocionales por parte de los establecimientos gastronómicos en la ciudad de Medellín; a partir de lo cual se establecen los objetivos de la investigación: Determinar el impacto del Influencer en las estrategias promocionales para el sector gastronómico de la ciudad de Medellín, para lo cual se pretende: a) Caracterizar los influencers que promocionan el sector gastronómico en Medellín; b) Identificar las redes sociales utilizadas por los influencers que promocionan el sector gastronómico en Medellín; c) Describir las herramientas de promoción empleadas por los influencers para el impulso del consumo de productos gastronómicos de la ciudad de Medellín; y d) Determinar el impacto del uso de influencers para la promoción en el sector gastronómico.

\section{Estado del Arte}

Desde inicios del actual siglo, se ha evidenciado que el desarrollo de la tecnología, particularmente del internet y de las redes sociales, han ejercido importante influjo en el modo de vida de la sociedad y en la manera en cómo las organizaciones deben hacer llegar sus marcas, productos y servicios a su público objetivo, replanteando sus estrategias promocionales y así beneficiarse en mayor medida de estas nuevas oportunidades para darse a conocer y posicionarse cada vez más en el mercado.

En primera instancia, Dhanesh y Duthler (2019), en su trabajo 'Gestión de relaciones a través de personas influyentes en las redes sociales' examinaron los efectos de la conciencia de los seguidores sobre el respaldo pagado a Influencers para la promoción o impulso de productos. Por medio de una encuesta, este estudio encontró que el reconocimiento de dicho respaldo por parte de los seguidores se relaciona con el reconocimiento de anuncios y a su vez se correlaciona con las intenciones de compra y el boca a boca. También descubrieron que la concientización sobre el respaldo pagado está correlacionada con la relación influencer-seguidor, que está asociada con las intenciones de compra y el voz a voz. Esta investigación permite saber, a quienes investigan, que la relación influenciador-seguidor no se ve necesariamente afectada aun cuando el seguidor reconoce que el contenido puede ser un anuncio pagado.

En segundo lugar, se presenta la investigación 'El Influencer: herramienta clave en el contexto digital en la publicidad engañosa' llevado a cabo por Gómez (2018), quien buscó analizar el nuevo panorama de la figura del influencer como elemento primordial en la estrategia Social Media de las organizaciones, y en el desarrollo de la publicidad engañosa y presentó la estrategia Influencers, motivos, utilización y su relación con la publicidad engañosa, además de analizar y exponer el vacío legal existente en la publicidad en línea 
mediante informes y estudios de expertos en el ámbito internacional para determinar a partir de casos, el vacío legal existente en el tratamiento de la publicidad digital aplicando una metodología mixta: conceptual/teórica y análisis de contenido. El aporte de Gómez (2018), permite conocer las razones y formatos utilizados en la estrategia de los Influencers, así como las ventajas e inconvenientes de la práctica comunicativa llevada a cabo por medio de ellos y la relación entre el Influencer y la publicidad engañosa dentro del marco legal que regula la actividad publicitaria tradicional y que puede generar vacíos cuando estas prácticas publicitarias se llevan a medios.

De igual manera, Gross y Wangenheim (2018) con su trabajo 'Los cuatro grandes del marketing influyente. Una tipología de los influyentes' abordaron el desafío que representa la correcta selección de una influencer, con el objetivo de caracterizar y presentar una tipología para los Influencers en cuatro grupos: fisgones, informadores, artistas e infotainers mostrando que la dinámica temporal y las transiciones de tipo influenciador basadas en la presencia social y la amplitud del dominio proporcionan una guía para que las empresas exploren con éxito a personas influyentes. Para llegar a tal clasificación en los tipos de Influencers se implementó un diseño de estudio exploratorio debido a la escasez de investigación sobre marketing de influencia, realizando entrevistas en profundidad que permitieron identificar las actividades, motivaciones, contenido y audiencia que los diferencia a cada uno de ellos. Con la clasificación presentada por los autores su puede establecer un punto de partida para identificar al tipo de influencer idóneo en la implementación de estrategias promocionales en plataformas digitales.

Por otra parte, el trabajo de Iglesias (2018), nombrado 'La eficiencia del marketing de Influencers en el ámbito gastronómico' cuyo objetivo fue establecer la eficiencia del marketing de Influencers en el ámbito gastronómico estudiando de manera específica el grado de fidelidad generado por los Influencers; especificando los elementos más relevantes de este tipo de marketing. Siendo un estudio de análisis de contenido de ocho personas de conocimiento gastronómico con más de 3000 seguidores en Instagram, delimitando las variables a número de seguidores, impacto de las publicaciones, estilo de las publicaciones, número de publicaciones promocionadas y tipo de acto publicitario.

Los resultados obtenidos por Iglesias (2018), muestran que no hay un consenso al momento de poder clasificar los Influencers con base a la cantidad de seguidores, siendo necesario que los organismos dedicados a la publicidad y marketing lleguen a un acuerdo para tal fin. Así mismo, se observó que no necesariamente a mayor cantidad de seguidores se genera mayor fidelidad lo que lleva a considerar otras variables adicionales al número de seguidores para valorar la calidad de un Influencers. Se identificó también que el tipo de promoción más habitual es la informativa y que los seguidores de los Influencers buscan la veracidad de un testimonio real basado en la experiencia.

Otro aporte de interés lo realiza Pedrayes (2018), en su artículo 'Los Influencers como modelo publicitario en redes sociales'. En él, buscaron 'conocer y profundizar la nueva publicidad basada en el uso de Influencers'. Se utilizó una metodología apoyada en la investigación documental con carácter descriptivo para comprender y conocer el uso de los líderes de opinión como forma publicitaria en las redes sociales, para explicar y describir el poder y la figura de los Influencers.

El trabajo de Pedrayes (2018), permite conocer cómo la sociedad está en constante conexión con las redes, buscando, compartiendo y experimentando contenido, es por esto que las redes sociales consiguen que la sociedad sea cada vez más sea 
digital y menos presencial, es así como se está en una continua adaptación digital, y que además las empresas están cambiando sus estrategias de venta adaptándose a este modelo más informatizado y digital; las compañías consiguen segmentar mejor su público conectándose de una manera más rápida y directa con el consumidor final; es por esto que es una de las estrategias más utilizadas debido a su gran efectividad.

Asimismo, el artículo 'El Influencer de moda como sujeto de relaciones públicas: recursos y herramientas' llevado a cabo por Villena (2018), el cual tuvo como objetivo proponer recursos y herramientas propias de las relaciones públicas 4.0, encaminadas a la correcta gestión de las relaciones con los Influencers por parte de los profesionales del sector. Siendo un estudio cualitativo, a través del estudio bibliográfico del concepto de Influencer como profesional de las relaciones públicas. Villena (2018), muestra como una de las acciones de relaciones públicas es la utilización de Influencers y como estos son los que mayor repercusión están teniendo en diferentes sectores dada la eficacia al momento de impactar al público con el que interactúan, y destaca como es necesario para las organizaciones que sus relacionistas públicos conozcan e implementen distintas herramientas para poder seleccionar de manera idónea y monitorizar el accionar de estos con la finalidad de obtener el máximo rendimiento.

Por otro lado, la investigación llevada a cabo por Fernández (2018), nombrada 'entre el uso y el abuso: la pérdida de credibilidad del marketing de Influencers', cuyos objetivos fueron analizar de la creciente utilización, efectos, eficacia y credibilidad de los Influencers como nuevo canal de comunicación; el estudio y profundización sobre las consecuencias negativas del marketing de Influencers. La metodología empleada fue un análisis bibliográfico, principalmente en publicaciones acerca de los Influencers como mecanismo al servicio publicitario de las marcas. Se utilizó una encuesta realizada a público con el fin de obtener información de uso y frecuencia de navegación en las diferentes plataformas, para así estudiar la influencia ejercida por cada uno de los Influencers, y la percepción individual acerca de la credibilidad y saturación del uso de estos como canales de comunicación.

El trabajo de Fernández (2018), evidenció que los usuarios valoran en gran medida la transparencia como uno de los factores clave en la comunicación. Esta atracción positiva despierta en los consumidores un sentimiento de confianza y seguridad, que produzca el suficiente interés y curiosidad por el usuario para querer conocer la marca, no solamente queriendo el incremento en las ventas sino produciendo engagement logrando clientes satisfechos y fieles a la marca.

Además, la investigación de Galvis \& Díaz (2017), con el título: 'Influencers, una estrategia de comunicación como posicionamiento de marca', donde su objetivo fue analizar la contribución de los Influencers en el posicionamiento de marca de las diferentes empresas en el sector gastronómico en la ciudad de Bogotá. La metodología empleada en esta investigación fue un enfoque cualitativo a partir de la observación conceptual de los influenciadores, los restaurantes a trabajar y las redes sociales, las herramientas implementadas fueron la encuesta, el focus group y las entrevistas a diferentes expertos en el tema.

La investigación Galvis \& Díaz (2017), permite entender un poco el mundo de los influenciadores en el sector gastronómico teniendo en cuenta cuales son los intereses de los usuarios, que quieren ver en los perfiles de las redes sociales, a su vez permite identificar los intereses más sobresalientes al utilizar una red social, y como los profesionales de la gastronomía identifican cada vez más que los influenciadores son medios para llegar a su público objetivo. 
A su vez, el trabajo de Arias y Márquez (2017), que lleva por título ‘La evolución de marketing de Influencers' y con el objetivo de comparar y demostrar el apogeo de las nuevas tendencias publicitarias en medios digitales frente a las tradicionales, bajo una metodología de diseño descriptivo, buscó la caracterización y descripción completa de la situación; igualmente se realizó una encuesta a 30 profesionales del sector de la publicidad. También usaron una metodología cuantitativa-cualitativa, ya que se buscó evaluar de una forma estadística la preferencia del sector publicitario que han realizado campañas utilizando influenciadores digitales.

Los hallazgos de Arias y Márquez (2017) permiten conocer el consumo de medios publicitarios, así como la evolución de la publicidad en los medios digitales ayudando a confiar en la hipótesis, por ellos planteada, de que la utilización de los Influencers no solo ha crecido en los últimos años, sino que crecerá de una forma considerable dado su auge y debido a su libertad creativa, generando un efecto dominó en el público objetivo.

Igualmente, en la investigación titulada "La Evolución Del Marketing Tradicional al De Influencia: Los Influencers" muestra como sus autoras: Nocito, Moya, Gutiérrez y Rothe (2017) buscaron demostrar la importancia de los Influencers en medios digitales como prescriptores de las marcas gracias a la evolución de la sociedad respecto a la captación de la publicidad en relación con las generaciones anteriores. Allí, las autoras plantean la hipótesis: ¿Son los Influencers una herramienta de marketing beneficiosa y productiva para las empresas en la actualidad? Las autoras acotaron y centraron su investigación en el sector de la moda y aplicaron y analizaron tres encuestas en cada uno de los ámbitos que influyeron en el desarrollo de su investigación: los consumidores, las empresas y los Influencers.

Los resultados obtenidos por Nocito, Moya, Gutiérrez y Rothe (2017), permiten comprender cómo el desarrollo tecnológico visto en las últimas décadas ha permitido potenciar cada vez más el internet y a su vez con la aparición de las redes sociales como estas se han constituido en una herramienta comunicativa cada vez mayor. Asimismo, como la conjunción de estos elementos ha constituido una gran oportunidad para las organizaciones permitiéndoles desarrollar nuevas estrategias de mercadeo más directas y efectivas pues les permiten influir en mayor medida las decisiones de compra de las nuevas generaciones.

La investigación llevada a cabo por Segarra, Hidalgo \& Rodríguez (2015), nombrada 'La gastronomía como industria creativa en un contexto digital', se centró en el análisis del contenido encontrado en los sitios web y redes sociales usadas por ocho restaurantes españoles galardonados con tres estrellas por la guía Michelin España Portugal, y cuyo objetivo principal fue el dar a conocer las estrategias comunicativas en línea aplicadas por dichos restaurantes y establecer la importancia que puede atribuirse a los recursos de la web al momento de contribuir a la consolidación de sus propias marcas así como la de la propia industria creativa gastronómica .

El trabajo de Segarra, Hidalgo \& Rodríguez (2015), permite evidenciar el éxito de la gastronomía no solo como el resultado de un ejercicio económico sino desde el punto de vista comunicativo y cómo este sector creativo de la gastronomía adquiere más participación en el mercado de la comunicación y cómo se desarrolla planteamientos estratégicos que posibiliten el acercamiento del sector a todo público haciéndolo más accesible y disponible para todo tipo de público y a su vez muestra que la integración de las redes sociales a dichas estrategias de comunicación.

Las investigaciones y artículos relacionados anteriormente abarcan material que provee fuentes de referencia acerca de temas directamente relacionados con el 
desarrollo de la investigación, como son: la publicidad, el marketing, redes sociales, entre otros; lo que les convierte en pertinentes para tal fin.

\section{Desarrollo conceptual}

Para efectos de esta investigación es necesario conocer los aspectos a tener en cuenta para el desarrollo de la investigación, como los son: la promoción, sus herramientas y estrategias, redes sociales como herramientas de promoción digital, el concepto de Influencers, los Influencers gastronómicos en la ciudad y el sector gastronómico.

\subsection{Promoción}

En primera instancia es necesario conocer el concepto de promoción para así entender cómo las organizaciones desde grandes a pequeñas deben desarrollar estrategias que permitan impulsar y/o potenciar las ventas de sus productos o servicios. Es entonces como Kotler, Cámara, Grande \& Cruz (2000, p. 98), afirman que la promoción, "es la herramienta del marketing, que incluye las distintas actividades que desarrollan las empresas para comunicar los méritos de sus productos y persuadir a su público objetivo para que compren". Así mismo, según Farber \& Bonta (2003, p. 44), la promoción es "el conjunto de técnicas integradas en el plan anual de marketing para alcanzar objetivos específicos, a través de diferentes estímulos y de acciones limitadas en el tiempo y en el espacio, orientadas a públicos determinados". Además, Pujol (1999, p. 277), la define como: "uno de los instrumentos fundamentales del marketing con el que la compañía pretende transmitir las cualidades de su producto a sus clientes, para que éstos se vean impulsados a adquirirlo; por tanto, consiste en un mecanismo de transmisión de información".

De las anteriores definiciones se considera como la más apropiada para el propósito de esta investigación la entregada por Pujol (1999), ya que en la enmarca en el contexto de la trasmisión de la información relacionada con usos, características y beneficios de la adquisición de un producto o servicio, algo muy propicio para llevar a cabo dentro de la actualidad digital donde predominan las redes sociales para tal fin, más allá de simplemente estimular mediante otras formas más tradicionales como cupones, degustaciones o descuentos.

\subsection{Mezcla promocional}

Según Amstrong \& Kotler (2003, p. 470), la mezcla de promoción "consiste en la combinación específica de herramientas de publicidad, promoción de ventas, relaciones públicas, ventas personales y marketing directo que la empresa utiliza para alcanzar sus objetivos de publicidad y marketing". Para Stanton, Etzel y Walker (2004, pp. 577-578), dicha mezcla promocional "es la combinación de ventas personales, publicidad, promoción de ventas y relaciones públicas de una organización”; y según el Diccionario de Marketing de Cultural (1999), es definida como un elemento del marketing mix de la empresa que sirve para informar al mercado y persuadirlo respecto a sus productos y servicios. 
Para el criterio de quienes investigan, la mezcla promocional se definirá entonces como la conjunción de las herramientas que permitirán transmitir al público objetivo la información relevante de productos y servicios y además persuadirlos para su adquisición y consumo.

A continuación, algunas de las definiciones que permiten abordar las diferentes herramientas de la mezcla promocional (Ver Cuadro 1).

Cuadro 1. Mezcla Promocional

\begin{tabular}{|c|c|}
\hline Autores & $\begin{array}{l}\text { Publicidad } \\
\end{array}$ \\
\hline $\begin{array}{l}\text { Según O'Guinn, Allen y } \\
\text { Semenik }(1999, \text { p. 6) }\end{array}$ & $\begin{array}{l}\text { La publicidad es un esfuerzo pagado, trasmitido por medios masivos de } \\
\text { información con objeto de persuadir. }\end{array}$ \\
\hline $\begin{array}{c}\text { Kotler y Armstrong } \\
(2003, \text { p. } 470)\end{array}$ & $\begin{array}{l}\text { Cualquier forma pagada de presentación y promoción no personal de ideas, } \\
\text { bienes o servicios por un patrocinador identificado. }\end{array}$ \\
\hline $\begin{array}{c}\text { Stanton, Walker y Etzel } \\
(2004, \text { p. } 569)\end{array}$ & $\begin{array}{l}\text { Una comunicación no personal, pagada por un patrocinador claramente } \\
\text { identificado, que promueve ideas, organizaciones o productos. }\end{array}$ \\
\hline Autores & $\begin{array}{l}\text { Venta Personal } \\
\end{array}$ \\
\hline $\begin{array}{l}\text { Lamb, Hair y McDaniel } \\
\text { (2002, p. } 543,544)\end{array}$ & $\begin{array}{l}\text { La comunicación directa entre un representante de ventas y uno o más } \\
\text { compradores potenciales, en un intento de relacionarse unos a otros en una } \\
\text { situación de compra. }\end{array}$ \\
\hline $\begin{array}{c}\text { Kotler y Armstrong } \\
(2003, \text { p. } 470)\end{array}$ & $\begin{array}{l}\text { Presentación personal que realiza la fuerza de ventas de la empresa con el } \\
\text { fin de efectuar una venta y cultivar relaciones con los clientes. }\end{array}$ \\
\hline $\begin{array}{l}\text { Richard L. Sandhusen } \\
(2002 \text {, p. } 522,523)\end{array}$ & $\begin{array}{l}\text { las ventas personales incluyen presentaciones de ventas cara a cara entre } \\
\text { intermediarios, clientes y prospectos. Generan relaciones personales a corto } \\
\text { y a largo plazo que agregan convicción persuasiva a las presentaciones de } \\
\text { ventas que relacionan los productos y servicios con las necesidades del } \\
\text { comprador. }\end{array}$ \\
\hline Autor & \begin{tabular}{|c|} 
Promoción de Ventas \\
\end{tabular} \\
\hline $\begin{array}{c}\text { Stanton, Etzel y Walker } \\
(2004, \text { p. } 637)\end{array}$ & $\begin{array}{l}\text { Los medios para estimular la demanda diseñados para completar la } \\
\text { publicidad y facilitar las ventas personales. }\end{array}$ \\
\hline $\begin{array}{l}\text { Kotler y Armstrong } \\
(2003, \text { p. } 470,476)\end{array}$ & $\begin{array}{l}\text { Los incentivos a corto plazo que fomentan la compra o venta de un } \\
\text { producto o servicio. }\end{array}$ \\
\hline $\begin{array}{c}\text { Romero (2003, p. 189- } \\
191) \\
\end{array}$ & $\begin{array}{l}\text { Un conjunto de ideas, planes y acciones comerciales que refuerzan la venta } \\
\text { activa y la publicidad, y apoyan el flujo del producto al consumidor. }\end{array}$ \\
\hline Autores & $\begin{array}{l}\text { Relaciones Publicas } \\
\end{array}$ \\
\hline $\begin{array}{l}\text { Cutlip, Center y Broom } \\
(2000,2001, \text { p. } 37)\end{array}$ & $\begin{array}{c}\text { Las relaciones públicas son la función directiva que establece y mantiene } \\
\text { relaciones mutuamente beneficiosas entre una organización y los públicos de } \\
\text { los que depende su éxito o fracaso. }\end{array}$ \\
\hline $\begin{array}{l}\text { Kotler, Armstrong, } \\
\text { Cámara y Cruz (2004, } \\
\text { p. } 542)\end{array}$ & $\begin{array}{c}\text { Son acciones que persiguen construir buenas relaciones con los } \\
\text { consumidores a partir de una publicidad favorable, la creación de una buena } \\
\text { imagen corporativa y evitando rumores, articulos periodisticos o } \\
\text { acontecimientos desfavorables, o haciendo frente a los mismos si llegan a } \\
\text { tener lugar. }\end{array}$ \\
\hline $\begin{array}{c}\text { Stanton, Walker y Etzel } \\
(2004, \text { p. } 643)\end{array}$ & $\begin{array}{l}\text { Una herramienta de administración destinada a influir favorablemente en las } \\
\text { actitudes hacia la organización, sus productos y sus politicas }\end{array}$ \\
\hline
\end{tabular}

Fuente: Elaboración propia (2020)

Es importante respecto en lo concerniente a la publicidad y para el interés de esta investigación centrarse en la definición dada por Stanton, Etzel \& Walker (2004), ya que no solo se centra en el impulso de bienes y servicios, sino que además tiene en cuenta la promoción de las organizaciones. Por su parte, en lo referente a la venta promocional se tomará en cuenta el concepto dado por Sandhusen (2002), ya que hace hincapié en las relaciones a corto y largo plazo con el público objetivo y en el uso de la convicción persuasiva en el proceso de venta basándose en las necesidades del comprador. En cuanto a la promoción de ventas se precisará como aquellas actividades comerciales planeadas que estimularon de manera activa el proceso de venta y generará una interacción más fluida entre el producto y el consumidor.

Finalmente, en lo entendido para las relaciones públicas y partiendo de los autores mencionados se entenderá entonces que las relaciones públicas son las actividades encaminadas al establecimiento de las relaciones favorables y duraderas desde la 
creación de una imagen corporativa buena para su público objetivo y que influya de manera positiva en la organización, sus políticas y productos.

\subsection{Estrategias de Promoción}

Aunque diferentes autores abordan la temática de las estrategias promocionales desde diferentes perspectivas, no es posible definir un estándar en la manera en que se clasifiquen, se planeen y se lleven a cabo pues estarán en función en el contexto y la actualidad bajo la cual la organización opere.

Por un lado, Burnett (1996), asevera que cualquier estrategia de promoción será exitosa partiendo no solo en la manera en que se lleva a cabo, sino que además dependerá también de la experiencia, el talento y la capacidad de quienes la ejecuten. Y clasifican a las estrategias en dos categorías: a) las estrategias de impulso orientadas a intermediarios y revendedores, cuyo esfuerzo va dirigido al almacenamiento, presentación al cliente y el mantenimiento del producto, y adecuada para los presupuestos bajos de promoción; b) las estrategias de atracción encaminada hacia el cliente final siendo más persuasiva y que genera una demanda más alta desde los diferentes canales que se manejen.

Mientras que Tellis \& Redondo (2002), muestran una variedad de estrategias promocionales enfocándose particularmente en ocho de ellas: Estrategias de Promoción para persuadir con información y argumentos, mediante estímulos emocionales, mediante testimonios, estrategias de promoción de ventas, del distribuidor, de fabricante, hacia el consumidor y con base en la comunicación integral.

En lo pertinente al interés de esta investigación se tomarán como referente las estrategias formuladas por Tellis \& Redondo (2002), centrándose específicamente en tres de ellas (Ver Cuadro 2).

Cuadro 2. Estrategias de Promoción

\begin{tabular}{|l|l|}
\hline $\begin{array}{l}\text { Estrategias de Promoción para } \\
\text { Persuadir con Información y } \\
\text { Argumentos }\end{array}$ & $\begin{array}{l}\text { De percepción } \\
\text { Para el Conocimiento } \\
\text { Para la Memorización o Recordación } \\
\end{array}$ \\
$\begin{array}{l}\text { De Argumentación: Comparativa, } \\
\text { Refutatoria, Preventiva, Enmarcación } \\
\text { y Argumento de Apoyo }\end{array}$ \\
$\begin{array}{l}\text { Estrategias de Promoción para } \\
\text { ersuadir mediante estímulos }\end{array}$ & $\begin{array}{l}\text { Explicativas } \\
\text { Asociativas }\end{array}$ \\
\hline $\begin{array}{l}\text { Estrategias de Promoción para } \\
\text { Persuadir mediante Testimonios }\end{array}$ & $\begin{array}{l}\text { De Credibilidad } \\
\text { De Atracción } \\
\text { De Transferencia de significados }\end{array}$ \\
\hline
\end{tabular}

Fuente: Tellis (2002). Diseñado y Adaptado por Amaya, Silva \& Rincón (2020)

El foco que se da a estos tres tipos de estrategias parte de que son las más utilizadas por los Influencers, objetos de esta investigación en la creación de contenido para el impulso de marcas y establecimientos del sector gastronómico.

\subsection{Red Social}

Partiendo de lo anterior se requiere entender el concepto de red social y comprender cómo esta puede constituirse en una herramienta de promoción digital, 
en tal sentido, Boyd y Ellison (2008, p. 211), afirman que una red social "es un servicio que permite a los individuos construir un perfil público o semipúblico dentro de un sistema delimitado, articular una lista de otros usuarios con los que comparten una conexión, y ver y recorrer su lista de las conexiones y de las realizadas por otros dentro del sistema". Por su parte, Celaya (2008, p. 92), señala que "Las redes sociales son lugares en Internet donde las personas publican y comparten todo tipo de información, personal y profesional, con terceras personas, conocidos y absolutos desconocidos"; y según Fowler y Christakis (2010, p. 27), "una red social está formada por un conjunto de personas entre las que existe una conexión por la que fluyen desde sentimientos hasta dinero".

A juicio de quienes investigan, las redes sociales son medios de comunicación en tiempo real, lo que busca es facilitar la comunicación entre grupos o comunidades con intereses en común, en donde se puede compartir mensajes, fotos, videos, opiniones, entre otros, haciendo que las personas estén interconectadas, además, hacen que su contenido llegue a un gran número de personas fácil y rápido.

Las redes sociales han establecido un antes y después en cuanto a la difusión de información de manera masiva, dado el gran alcance que tienen y el impacto que generan en la sociedad. Además, permiten una mayor interacción y dinamismo, ya que son usadas conjuntamente por individuos y empresas. Dada la acogida de las redes sociales y su gran crecimiento a lo largo de estos últimos años, han surgido gran variedad de ellas no solo para ámbitos social o general, sino también, empresarial para efectos de negocio y marketing. Las organizaciones han logrado conseguir a través de estas redes sociales una comunicación más efectiva y fluida ya que con ellas logran llegar a grupos específicos de interés y conseguir una mayor integración e interacción con sus clientes, lo que ha facilitado aumentar la proyección y el posicionamiento de las empresas, las marcas, los productos y los servicios e incluso llegar a ser utilizadas en campañas publicitarias. Asimismo, y aunque durante los últimos años, según Celaya (2008) en Herrera (2012):

"Su crecimiento ha tendido a disminuir para dar espacio a nuevas plataformas especializadas en áreas temáticas, las redes sociales seguirán siendo uno de los medios de comunicación masiva más importantes, debido a sus características y a las opciones que ofrecen." (p. 1)

La efectividad de los medios tradicionales en los que la publicidad es emitida ha disminuido por la saturación audiovisual que se percibe ya que en muchas ocasiones no logra llegar a nichos específicos y además resulta muy costosa.

\subsection{Influencers}

Por otra parte, se ha evidenciado una notable tendencia a que los consumidores han comenzado a creer más en las opiniones de otras personas a simplemente fiarse de la publicidad y promoción que perciben en los diferentes medios y es allí donde muchas de estas personas convergen en las diferentes redes sociales no solo a expresar sus opiniones sino a conocer la de las demás personas. Y es a partir de esta tendencia donde aparecen en escena algunas personas que han creado sus propios blogs donde comenzaron a hacer reseñas de productos y servicios, especializándose 
en ellos, convirtiéndose en comunicadores natos, siendo espontáneos y naturales en sus opiniones y que empezaron a generar grupos de seguidores que los escuchan y les dan valor a sus intervenciones. Estas personas con un potencial alto de difusión e influencia se les conoce como Influencers, y se pueden definir como según lo expresa el Diccionario LID de Marketing Directo e Interactivo (2015), el influenciador es quien transmite información de productos, servicios, gracias al auge de las redes sociales, de cualquier tema de actualidad, normalmente se especializan en un tema específico e interactuar con otros usuarios compartiendo sus opiniones, pensamientos e ideas.

Se puede entender este concepto también, en atención a las consideraciones de Dorantes (2012), como:

"aquellas personas que consumen, generan información y que normalmente hablan de un tema en específico. Por lo general, cuando interactúan y comparten sus opiniones, ideas o reflexiones con sus seguidores, estos están más que dispuestos a adoptar y compartir su mensaje." (Dorantes, 2012, p. 1)

Mientras tanto, Almeida (2017, p. 13) afirma que "un Influencers es un profesional que, debido a sus conocimientos y pericia en un sector, se convierte en un fiable prescriptor de un producto o servicio para el público en general".

Para el particular fin de esta investigación quienes investigan definen al Influencers como aquella persona que, gracias a su conocimiento, habilidad en un tema específico y alta notoriedad pública, genera contenido relacionado a un producto o servicio y que por medio de su opinión ejerce un papel activo de influencia sobre la comunidad con la que se relaciona.

Partiendo de lo anteriormente definido es entonces donde las empresas y sus marcas se han dado cuenta del potencial tan grande que tienen estos Influencers y como podrían ser usados en el desarrollo de sus nuevas estrategias comunicativas y poder llegar de manera más directa a grupos específicos y minimizando los altos costos de la publicidad tradicional, de la mano de inversión tecnológica que posibilite tener una mayor perspectiva de crecimiento económico.

Diariamente en internet se encuentran millones de personas buscando información de aquellos sitios que desean conocer y dado el auge de las redes sociales, acuden a ellas para conocer, de primera mano, experiencias previas de otras personas que ya hayan estado allí. Y es entonces, en las redes sociales, donde dentro de las muchas opiniones que pueden encontrarse, están las de quienes se han convertido para muchos en la primera fuente de información fiable para encontrar opiniones positivas o negativas, los Influencers, y hacerse a una primera impresión aún sin visitar el sitio y es el primer filtro que podrá lograr que las personas se animen a visitar.

\subsection{Sector Gastronómico}

En el ámbito gastronómico, los restaurantes y sitios de comida informal de la ciudad de Medellín, en el transcurso del último año, han gozado de un gran apogeo, producto de la credibilidad de los Influencers en este sector, particularmente, gracias a la aparición en escena del que es considerado el Influencers más reconocido en este ámbito no solo a nivel ciudad, sino a nivel país, Tulio Zuloaga (1970). Gracias a él, un gran número de restaurantes e incluso sitios de comida callejera han tenido la 
oportunidad de ser visitados por él y luego ser reseñados en los diferentes perfiles usados por él en redes sociales.

Fruto de estas publicaciones, cientos de sitios, que antes eran desconocidos para muchos, se han dado a conocer dentro del sector gastronómico de la ciudad y gracias a la credibilidad de la que goza este Influencers, estos lugares han experimentado un aumento en el flujo de sus clientes y contribuyendo incluso a la sostenibilidad de sus negocios y en algunos casos logrando mejorar su posicionamiento en el sector.

Por otra parte, este Influencers ha sido el creador de eventos que han revolucionado el sector, por una parte, estimulando subsectores como el de hamburguesas, el de pizzas y el de sushi logrando que los restaurantes den a conocer sus mejores recetas además impulsando a que logren innovar con nuevas recetas que dinamicen sus negocios que puedan hacerse más atractivos no solo para sus clientes habituales sino también para sus clientes potenciales. Además, este tipo de eventos ha generado en los comensales la curiosidad de conocer nuevos sitios y recetas y así permitirles conocer muchos más lugares en los que puedan disfrutar una variedad más amplia de platos más allá de lo que tradicionalmente pueden conocer.

Además, en el aspecto económico, esta clase de eventos han generado ventas millonarias con cifras nunca antes vistas en estos sectores, llegando a vender aproximadamente \$14.133.000.000 (Zuloaga, 2018a) en solo 7 días de duración del evento Burger Master, así como más de 10 mil millones de pesos con el Sushi Master (Zuloaga, 2018b) y \$5.807.000.000 con el Pizza Master (Zuloaga, 2018c), todos estos realizados durante el año 2018.

Estas cifras son evidencia del gran impacto que puede generar un Influencer en el sector gastronómico, y como este puede ayudar al crecimiento económico de un sector y, además, es una muestra de la gran influencia que pueden ejercer sobre miles o incluso sobre millones de personas. Demostrando así, que este tipo de publicidad es efectiva, solo si los dueños de los establecimientos trabajan en conjunto para fortalecer su sector y poder crear planes estratégicos, a partir de estas experiencias, que les permitan la sostenibilidad de sus negocios.

\subsection{Influencers Gastronómicos}

En la escena local de Influencers gastronómicos se logran encontrar cientos de cuentas que se dedican exclusivamente a la comida, algunas direccionadas a la sana alimentación, mientras que otra a repostería, y muchas de ellas a la comida gourmet u otras especialidades, la mayor parte de estas cuentas lo que quieren lograr es ser guía para los nuevos sitios y las nuevas tendencias. Entre ellos se destaca @parasaborearse con algo más de 60.100 seguidores, así como @saborenlamesa con unos 48.000 seguidores, también se encuentra@quehacermedellín con unos 126.000 seguidores, así como@dondecomer con aproximadamente unos 98.000 seguidores, además, esta @eat_medellin que tiene unos 35.700 seguidores, esta @medellin_food_guide con 115.000 seguidores, @gastronomiacasual cuenta con 27.200 seguidores, también se tiene a@medellineats con67.900 seguidores y finalmente @Tuliorecomienda siendo la cuneta más seguida con 417.000 seguidores, todos estos nada más en Instagram. Entrar en sus perfiles es una invitación a degustar los mejores sabores que se puedan encontrar en la ciudad ya que a través de sus cuidadosas fotografías y sus detalladas y creativas reseñas logran cautivar la atención y deseos de quienes visitan estos perfiles. 


\section{Metodología}

La presente investigación es de tipo descriptivo ya que según Tamayo \& Tamayo (2003, p. 46) "comprende la descripción, registro, análisis e interpretación de la naturaleza actual, y la composición o proceso de los fenómenos. El enfoque se hace sobre conclusiones dominantes o sobre grupo de personas, grupo o cosas, se conduce o funciona en presente". Según lo definido anteriormente, esta investigación tomará entonces una forma descriptiva ya que su propósito será determinar el impacto de los Influencers en las estrategias promocionales para el sector gastronómico de la ciudad de Medellín.

Quienes investigan establecen el diseño de esta investigación como no experimental, transeccional descriptivo y de campo. Según Hernández, Fernández y Baptista (2014), este tipo de estudio no experimentales se llevan a cabo sin manipular deliberadamente las variables, observando fenómenos tal como se dan en su contexto natural, con el propósito de analizarlos; además, no se genera ninguna situación, sino que se observan situaciones ya existentes, no provocadas intencionalmente en la investigación por quien la realiza.

Por otra parte, en los diseños transeccionales, según Hernández, Fernández y Baptista (2014), la recopilación de información se hará en un único momento y el propósito será analizar las variables y su incidencia e interrelación en un único momento. Al tiempo que, los diseños de campo según Arias (2012), están centrados en los datos obtenidos en la realidad es donde ocurren; a partir de fuentes vivas o directas las cuales quedan representadas en la población.

La presente investigación está conformada por tres poblaciones según se describe a continuación:

La población 1: se estableció a partir de cuentas de Influencers del sector gastronómico de la ciudad de Medellín en la red social Instagram; las cuales fueron seleccionadas en atención al muestreo intencional o por conveniencia considerando el número de seguidores, y sistematizadas a partir de la observación directa mediante entrevistas cualitativas.

La población 2: quedó determinada por establecimientos gastronómicos de la ciudad de Medellín, los cuales han sido seleccionados en atención al muestreo intencional o por conveniencia, considerando su participación en eventos gastronómicos, los cuales fueron abordados a partir de las entrevistas a propietarios y/o gerentes de dichos establecimientos.

La población 3: se establece con base en los habitantes de la ciudad de Medellín, los cuales según el Dane (2019), conforman 2.508.452 personas mayores de 18 años, representados por una muestra probabilística al azar de 246 sujetos en atención la fórmula de poblaciones finitas que explican Munch y Ángeles (1995), aplicando posteriormente el Factor de Corrección de Hernández, Fernández y Baptista (2003); lo cuales serán abordados mediante un cuestionario de selección múltiple y multivariado, además, entrevistas a Influencers del mismo sector y a propietarios y/o gerentes de establecimientos gastronómicos de la ciudad de Medellín.

El diseño de las muestras de las poblaciones 1 y de la población 2 se consideró como no probabilística, basándose en una muestra por conveniencia, que permite a quienes investigan, seleccionar de manera intencional las fuentes vivas o directas. En cuanto a la población 3 se consideró como probabilística, así como según lo define 
Hernández, Fernández \& Baptista (2014), ya que la posibilidad de selección es la misma por parte de los elementos que la componen.

Se procede a realizar el análisis descriptivo inferencial en atención a los objetivos planteados para el presente estudio.

\section{Análisis y discusión de resultados}

Una vez aplicados los instrumentos de entrevistas a Influencers y a propietarios y/o administradores de establecimientos gastronómicos, así como, la aplicación de la encuesta al público en general de la ciudad de Medellín, se encontró que:

Para dar respuesta al primero de los objetivos planteados caracterizar los Influencers que promocionan el sector gastronómico en Medellín y una vez aplicados los instrumentos diseñados, se encontró la siguiente caracterización (Ver Tabla 1).

Tabla 1. Caracterización Influencers

\begin{tabular}{|c|c|c|c|c|}
\hline Caracterización & Influencer 1 & Influencer 2 & Influencer 3 & Influencer 4 \\
\hline Genero & Femenino & Hombre & Femenino & Femenino \\
\hline Profesión & $\begin{array}{c}\text { Comunicadora Grafica } \\
\text { Publicitaria }\end{array}$ & $\begin{array}{c}\text { Profesional en } \\
\text { Formación - Mercadeo }\end{array}$ & Abogada & $\begin{array}{c}\text { Profesional en } \\
\text { Mercadeo }\end{array}$ \\
\hline Redes Sociales & Facebook - Instagram & Instagram & Facebook - Instagram & Facebook - Instagram \\
\hline Red social más utilizada & \multicolumn{4}{|c|}{ Instagram } \\
\hline Tipo de Contenido & Foto, Video y Reseña & Foto y Reseña & Foto, Video y Reseña & Foto y Reseña \\
\hline Número de Seguidores & 126.000 & 115.000 & 97.900 & 21.100 \\
\hline Numero de Publicaciones & 4.289 & 269 & 3.603 & 1.190 \\
\hline Estrategias Promocionales & \multicolumn{4}{|c|}{ Información, Persuasión por emociones y Persuasión por Testimonios } \\
\hline Herramientas Promocionales & $\begin{array}{c}\text { Concursos } \\
\text { Lanzamientos de } \\
\text { producto } \\
\text { Descuento } \\
-2 \mathrm{x} 1 \\
\text { Eventos } \\
\end{array}$ & $\begin{array}{c}\text { Concursos } \\
\text { Lanzamientos }\end{array}$ & $\begin{array}{c}\text { Concursos } \\
\text { Lanzamientos de } \\
\text { producto } \\
\text { Descuento } \\
2 \times 1\end{array}$ & $\begin{array}{c}\text { sorteos } \\
\text { Inauguraciones } \\
\text { Descuentos } \\
\text { Lanzamientos de } \\
\text { productos }\end{array}$ \\
\hline Contenido Auspiciado & $\mathrm{Si}$ & No & $\mathrm{Si}$ & $\mathrm{Si}$ \\
\hline
\end{tabular}

Fuente: Elaboración propia (2020)

Como puede evidenciarse en la Tabla 1, el común de los influencers se enmarcan en asocio con formación profesional en el ámbito de las comunicación, el marketing y profesiones asociadas, se podría considerar que una de las mayores fortalezas de estos es su capacidad de oratoria empleada a partir de estrategias como la información, la persuasión por emociones y a partir de testimoniales; estrategias que han sido descritas por Tellis \& Redondo (2002); los influencers se proyectan de manera simultánea en diversas redes sociales, siendo Instagram la plataforma donde operan preponderantemente, se pudo observar entre las herramientas promocionales que emplean destacan los concursos, lanzamientos de productos y descuentos; empleando de igual manera sus redes para la promoción de eventos.

Ahora bien, en lo que se refiere al segundo objetivo planteado identificar las redes sociales utilizadas por los Influencers que promocionan el sector gastronómico en Medellín, las entrevistas permitieron identificar que de manera predilecta la RRSS más usada por ellos es Instagram, debido particularmente al impacto visual que puedan generar a través de ella y de la respuesta lograda a cada publicación por parte de sus 
seguidores. Esta preferencia de uso resulta acertada por parte de los influenciadores ya que, gracias a la aplicación de la encuesta y entrevistas, es precisamente esta misma RRSS la que se muestra como preferencia de uso por parte del público en general ver (Gráfica 1), así como a su vez es la más usada por los establecimientos gastronómicos para sus estrategias de marketing digital permitiéndoles mayor interacción con sus clientes actuales y potenciales.

Gráfica 1. Red Social más utilizada por el público

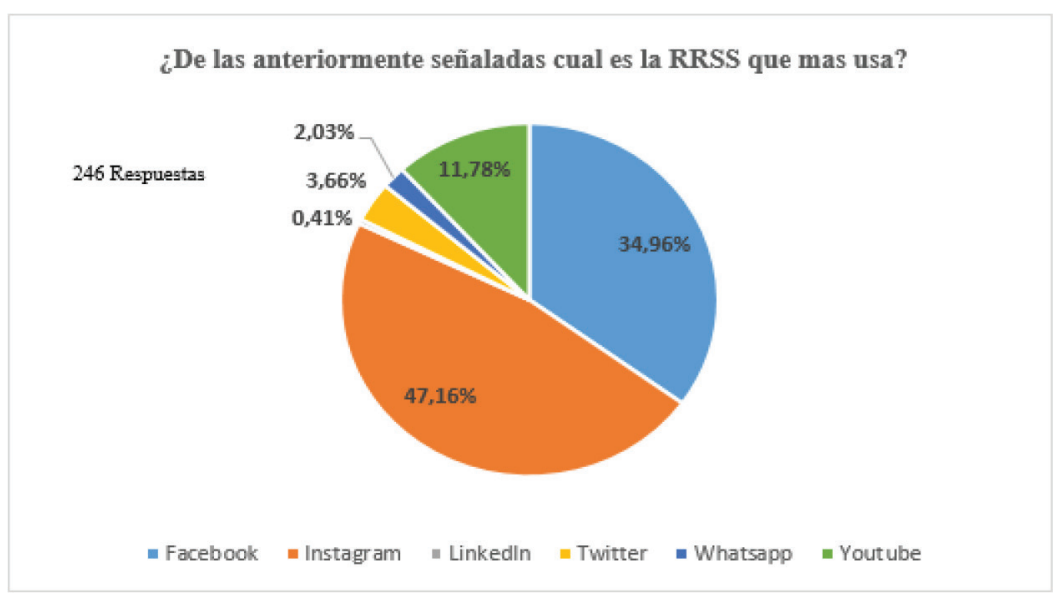

Fuente: Elaboración propia (2020)

En la Gráfica 1, se puede observar cómo los usuarios de los servicios gastronómicos en Medellín, señalan que la red social más empleada es Instagram, seguida de Facebook, YouTube; Twitter y WhatsApp, parece pertinente observar que esta última establece un contacto más directo con los usuarios de los servicios, posibilitando una retroalimentación o feedback directo en el contexto promocional; lo anterior se corresponde con las redes sociales que están siendo empleadas por los influencers para impactar a los usuarios de servicios gastronómicos.

Para responder al objetivo de describir las herramientas de promoción empleadas por los Influencers para el impulso del consumo de productos gastronómicos de la ciudad de Medellín, se evidencia mediante las entrevistas efectuadas tanto a los establecimientos gastronómicos como a los propios Influencers que dentro de las herramientas promocionales más usadas se destacan los concursos, los eventos y ofertas pague uno lleve dos (2x1), sorteos, lanzamientos de carta y relanzamiento de productos, siendo estos particularmente beneficiosos para el reconocimiento y recordación de la marca, la adquisición de seguidores y la interacción que con ellos se pueda generan en las RRSS. En tal sentido, se las herramientas empleadas se corresponden con las estrategias planteadas por Tellis \& Redondo (2002), para: a) persuadir con información y argumentos, b) persuadir mediante estímulos emocionales, y c) para persuadir con testimonios.

Finalmente, para determinar el impacto del uso de Influencers para la promoción en el sector gastronómico, la aplicación de las entrevistas permitió encontrar que los propietarios y/o gerentes de los establecimientos objeto de estudio convergen en que la utilización de los servicios ofrecidos por los diversos Influencers, ha posibilitado la 
afluencia de clientes en sus negocios aumente considerablemente; así como la contribución al cumplimento de las metas por ellos establecidas en lo que a ventas se refiere y que el alcance de dichas publicaciones se logre directamente sobre personas que comparten intereses gastronómicos; todo esto en comparación con lo que podrían lograr con otros medios tradicionales, ya que estos han considerado que la primera opción sean los Influencers al momento de publicar cualquier tipo de contenido cuya finalidad sea el impulso o promoción bien sea de la marca o de sus productos. Esto se corresponde a lo planteado por Villena (2018), quien señala que el empleo de Influencers como una acción de relaciones públicas establece mayor repercusión y eficacia al momento de alcanzar a los públicos con la finalidad de alcanzar el máximo rendimiento.

\section{Conclusiones}

Esta investigación lleva a concluir que en cuanto a la formación profesional de los influencers estos podrían no estar vinculados directamente a estudios gastronómicos; sin embargo, destaca el tipo de formación profesional con predominio en áreas de comunicación y marketing; al tiempo que el éxito del desempeño del 'influencer gastronómico' no depende de su formación profesional, evidenciándose como algunos iniciaron esta actividad por hobby y con el transcurrir del tiempo la experiencia y conocimiento adquiridos conlleva al desarrollo de esta actividad en un nivel profesional.

Otro aspecto a destacar es lo relacionado con las estrategias promocionales, ya que en las cuentas de los influencers objetos de estudio, se encontró que las estrategias de información, de persuasión por emociones y de persuasión por testimonios están más alineadas al tipo de contenido que puede generarse a través de las redes sociales empleadas por ellos.

Por otra parte, se establecieron que las herramientas más utilizadas son: los concursos y las ofertas pague uno lleve dos (2x1), las cuales generan resultan particularmente efectivas para captar más clientes, aumentar sus ventas, así como la cantidad de personas que puedan reconocer la marca y a su vez generar mayor recordación de la misma; ya que con este tipo de herramientas logran mayor alcance de su contenido y de sus cuentas e interacciones que puedan crear con quienes les siguen.

Si bien en la actualidad existe una gran variedad de RRSS para el caso del sector gastronómico resulta particularmente sensible al empleo de la red social Instagram, ya que permite visualmente ser más atractiva para los seguidores al momento de publicar contenido de productos gastronómicos; así pues entre los hallazgos de esta investigación se precisa que en esta red social el contenido adquiere un mayor alcance.

El éxito de los Influencers radica en la credibilidad que generan y lograr ser referentes por la utilización y demostración de una gran variedad de productos, al igual que por su opinión y consejos, pues se convierten en guías que a su vez aclaran las dudas que sus seguidores puedan tener sobre algún producto en el cual estén interesados. Estos Influencers terminan por convertirse en líderes de opinión y quienes los siguen a su vez los alientan a crear más contenido para dar a conocer al mundo más productos, más servicios, más marcas y con estos generar conocimiento de marca y crear también deseos de adquirirlos en un ciclo que parece no tener fin. Las diferentes marcas, grandes o no, se han dado cuenta de esto y han comenzado a utilizar a estos 
Influencers cada vez más pues por medio de ellos logran dar a conocer en mayor medida sus productos a nichos de mercado de manera más eficiente y con costos mucho menores.

Ciertamente, los influencers han constituido un medio más eficiente para lograr un mayor reconocimiento y alcance de las marcas, según los reconocen los propietarios y/o administradores de los restaurantes que fueron objetos de esta investigación, ya que logran en menos tiempo y a un menor costo el impacto que quieren generar sobre su sector en comparación con los medios que anteriormente se utilizaban. Sin embargo, estos restaurantes dentro de su gestión de resultados no mostraron la utilización de algún indicador particularmente diseñado para medir el impacto que estos influencers pudieran lograr y lo soportan con las cifras obtenidas en otros indicadores como sus ventas y flujo de clientes en sus establecimientos, todo esto en conjunción con las cifras que luego de cada publicación les son compartidas por los influencers donde se revelan el alcance de cada una de estas tenía en la red.

Finalmente, se concluye que el trabajo conjunto entre establecimientos e influencers ha llevado a que el sector gastronómico en la ciudad de Medellín se vea potenciado en cuanto el reconocimiento de las marcas y su posicionamiento en él y que al mismo tiempo ha permitido que la población de la ciudad de Medellín identifique la gran variedad de restaurantes existentes en la ciudad y a su vez la diversidad gastronómica que en ella se puede disfrutar desde lo más pequeños y básicos negocios hasta los más sofisticados y elegantes restaurantes, además, se ha visto estimulada la creación de emprendimientos en el sector y también ha constituido la oportunidad de que algunas personas quieran desarrollar actividades en torno a su gusto por la comida, en el caso de los influencers, por lo cual se concluye que el impacto del Influencer en las estrategias promocionales para el sector gastronómico de la ciudad de Medellín es altamente favorable.

\section{Bibliografía}

ABC. (8 de Junio de 2018). www.abc.es. Obtenido de www.abc.es: https://www.abc.es/play/ television/noticias/abci-anthony-bourdain-icono-television-estados-unidos-201806081437_ noticia.html

Almeida, R. (2017). Influencers la nueva tendencia del marketing online. En R. Almeida, Influencers la nueva tendencia del marketing online (pág. 13). Barcelona: Editorial Base.

Amstrong \& Kotler, P. (2003). Fundamentos de Marketing, Sexta Edición. En P. K. Amstrong, Fundamentos de Marketing, Sexta Edición (pág. 470). México: Pearson/Prentice Hall.

Arias, F. G. (2012). El proyecto de Investigación. Introducción a la metodología científica sexta edición. En F. G. Arias, El proyecto de Investigación. Introducción a la metodología científica sexta edición, p. 31. Caracas: Episteme.

Arias \& Márquez, M. (2017). Evolución del Marketing de Influencers. Obtenido de https://idus. us.es/xmlui/handle/11441/63284

Bernal, C. A. (2010). Metodología de la investigación. En C. A. Bernal, Metodología de la investigación, p. 113. Bogotá: Prentice Hall.

Booth \& Matic, N. (2011). Mapeo y aprovechamiento de personas influyentes en las redes sociales para moldear las percepciones de la marca corporativa. (G. E. Norma, Ed.) Obtenido de Mapping and leveraging influencers in social media to shape corporate brand perceptions: https://www.emeraldinsight.com/doi/abs/10.1108/13563281111156853

Boyd \& Ellison. (2008). Social Network sites: Definition, history and scholarship. Journal of Computer-Mediated Communication, 211. 
Burnett, J. (1996). Promoción, Conceptos y Estrategias. Colombia: MCGraw Hill.

Castellano \& Del Pino, A. (2015). La comunicación publicitaria con influencers. Obtenido de dialnet.unirioja.es: https://dialnet.unirioja.es/servlet/articulo?codigo $=5159613$

Celaya. (2008). La empresa en la web 2.0. La web 2.0, 92.

Cohen, L., Manion, L., \& Morrison, k. (2003). Métodos de Investigación en Educación. Londres: Routledge Falmer.

Conde, K. (2018). Estrategias de los Digital Influencers y su incidencia en el posicionamiento de las MYPES textiles de la ciudad de Trujillo, 2018. CASO: KTC Ropa y accesorios. Obtenido de http://repositorio.ucv.edu.pe/handle/UCV/17866

Creswell, J. W. (2009). Diseño de la Investigación. Los Angeles: Sage Publications.

Dhanesh \& Duthler, G. (30 de 03 de 2019). Gestión de relaciones a través de personas influyentes en las redes sociales. Revista Public Relations Review disponible online $30 \mathrm{March}$ 2019. Elsevier. doi:Dhanesh, G. S. (2019). Gestión de relaciones a través de personas influyentes en las redes sociales. Obtenido de www.sciencedirect.com: https://www.sciencedirect.com/science/article/abs/pii/S0363811118305216\#!

Diccionario de marketing /Cultural, S. A. (1999). Diccionario de marketing/Cultural, S. A. España: Cultural.

Directo, D. M. (17 de 08 de 2015). Interactivo, Diccionario LID de Marketing Directo e. Obtenido demarketing.directo.com:https://www.marketingdirecto.com/diccionario-marketing-publicidadcomunicacion-nuevas-tecnologias/influenciadores

Dorantes, H. H. (27 de 07 de 2012). Influenciadores ¿Quiénes son realmente? Merca2.0, p. 1. Obtenido de merca20.com: https://www.merca20.com/influenciadores-quienes-son-realmente

Farber \& Bonta, P. (2003). Preguntas sobre Marketing y Publicidad. En P. B. Farber, Preguntas sobre Marketing y Publicidad, p. 44. Grupo Editorial Norma.

Fernández, M. D. (04 de 06 de 2018). Entre el uso y el abuso: la pérdida de credibilidad del marketing de influencers. Obtenido de http://uvadoc.uva.es/handle/10324/34068

Fowler \& Christakis, N. (2010). Conectados. En N. A. Fowler, Conectados, p. 27. Madrid: Editorial Taurus.

Galvis \& Diaz, G. (2017). Influencers, una estrategia de comunicación como posicionamiento de marca. Bogotá. Obtenido de https://repository.usta.edu.co/bitstream/handle/11634/10152/ D\%C3\%ADaz2017.pdf?sequence=1

Gómez, B. (Junio de 2018). El Influencer: Herramienta clave en el contexto digital de la publicidad engañosa. Obtenido de www.redalyc.org: https://www.methaodos.org/revista-methaodos/index.php/methaodos/article/viewFile/212/359

Gross \& Wangenheim, J. (2018). Los cuatro grandes del marketing influyente. Una tipología de los influyentes. Obtenido de The Big Four of Influencer Marketing. A Typology of Influencers: https://papers.ssrn.com/sol3/papers.cfm?abstract_id=3230687

Hernández, R., Fernández, C. \& Baptista, M. d. (2003). Metodología de la Investigación Quinta Edición. México: McGRAW-HILL.

Hernández, R., Fernández, C. \& Baptisa, M. d. (2014). Metodología de la Investigación sexta edición. México: McGraw Hill.

Herrera, H. H. (09 de 02 de 2012). Las redes sociales: una nueva herramienta de difusión. Las redes sociales: una nueva herramienta de difusión, 1.

Höchsmann, F. (2014). Manual para el mejor servicio de calidad en hoteles y restaurantes. En F. Höchsmann, Höchsmann, Frank, p. 49. Books on Demand.

Iglesias, Y. (25 de Febrero de 2018). La eficiencia del marketing de influencers en el ámbito gastronómico. Obtenido de reunir.unir.net: https://reunir.unir.net/handle/123456789/7396

Kotler P., Cámara, D., Grande, I. \& Cruz, P. (2000). Dirección de Marketing. En C. D. Kotler Philip, Dirección de Marketing, p. 98. Prentice Hall.

McMillan, J., \& Schumacher, S. (2001). Investigación en Educación: Una Introducción Conceptual. New York: Addison Wesley Longman. 
Melendez \& Cañez, J. (2010). La cocina tradicional regional como elemento de identidad y desarrollo local. Obtenido de http://www.scielo.org.mx/pdf/estsoc/v17nspe/v17nspea8.pdf

Munch, L., \& Angeles, E. (1995). Metodos y Tecnicas de Investigacion. México: Editorial Trillas.

Nocito, Moya, Gutiérrez \& Rothe, M. (2017). La evolución del marketing tradicional al de influencia: Los influencers. Obtenido de https://www.unav.edu/ documents/4889803/13079787/62_Orvalle+-+La+evoluci\%C3\%B3n+del+Marketing+tradicional+al+de+influencia+Los+influencers.pdf/5d9ee093-ff02-1d1a-c20f-714e021e9c5d

Pedrayes, M. (25 de Febrero de 2018). Los influencers como modelo publicitario en redes sociales. Obtenido de uvadoc.uva.es: http://uvadoc.uva.es/handle/10324/31088

Pujol, B. (1999). Diccionario de Marketing, de Cultural S.A. En P. Bengoechea, Diccionario de Marketing, de Cultural S.A, p. 277. Madrid: Cultural.

Rodríguez, A. (2017). El nacimiento de un nuevo marketing: influencers en España año 2017; cambio de hábito de consumo en España entre bombres y mujeres influenciados a través de las redes. Obtenido de https://uvadoc.uva.es/bitstream/10324/24861/1/TFG-N.\%20759.pdf

Sandhusen, R. L. (2002). Mercadotecnia. México: Cecsa (Compañia Editorial Continente).

Segarra; Hidalgo \& Rodriguez, J. (23 de 06 de 2015). La gastronomía como industria creativa en un contexto digital. Análisis de webs y redes sociales de los restaurantes españoles con estrella Michelin. Madrid: Ad Comunica. Obtenido de http://www.adcomunicarevista.com/ ojs/index.php/adcomunica/article/view/254

Stanton, M. J.; Stanton, W. J.; Walker, B. J. (2004). Fundamentos de marketing, 13a Edición. En Stanton, \& E. Walker, Fundamentos de marketing, 13 a Edición, pp. 577-578. Mexico: Hill Interamericana.

Tamayo \& Tamayo, M. (2003). Proceso de Investigación Científica. En M. T. Tamayo, Proceso de Investigación Científica, p. 46. México: Limusa S.A.

Tellis \& Redondo, G. (2002). Estrategias de Publicidad y Promoción. En G. J. Redondo, Estrategias de Publicidad y Promoción, pp. 282-283. Madrid: Pearson Educación S.A.

Travelchannel. (18 de 03 de 2019). www.travelchannel.com. Obtenido de www.travelchannel. com: https://www.travelchannel.com/shows/bizarre-foods

Villena, E. (2018). El influencer de moda como sujeto de relaciones públicas: recursos y herramientas. Obtenido de http://revistas.udc.es/index.php/REDMARKA/article/view/ redma.2018.01.022.4938

Zuloaga, T. (12 de Mayo de 2018a). Tulio Recomienda Guía de los Mejores Restaurantes. Obtenido de Tulio Recomienda Guía de los Mejores Restaurantes: https:/tuliorecomienda. wordpress.com/2018/05/12/quienes-fueron-los-ganadores-del-burger-master/

Zuloaga, T. (11 de Septiembre de 2018b). Tulio Recomienda Guía de los Mejores Restaurantes. Obtenido de Tulio Recomienda Guía de los Mejores Restaurantes: https://tuliorecomienda. wordpress.com/category/sushi-master/

Zuloaga, T. (1 de Noviembre de 2018c). Tulio Recomienda Guía de los Mejores Restaurantes. Obtenido de Tulio Recomienda Guía de los Mejores Restaurantes: https://tuliorecomienda. wordpress.com/category/1-1-pizza-master/ 